\title{
Identification of Fractional-Order Transfer Functions Using a Step Excitation
}

\author{
Luiz Antonio Jacyntho, Marcelo Carvalho Minhoto Teixeira, Member, IEEE, Edvaldo Assunção, Rodrigo Cardim, \\ Roberto Kawakami Harrop Galvão, Senior Member, IEEE, and Sillas Hadjiloucas, Senior Member, IEEE
}

\begin{abstract}
This brief proposes a new method for the identification of fractional-order transfer functions based on the time response resulting from a single step excitation. The proposed method is applied to the identification of a 3-D RC network, which can be tailored in terms of topology and composition to emulate real-time systems governed by fractional-order dynamics. The results are in excellent agreement with the actual network response, yet the identification procedure only requires a small number of coefficients to be determined, demonstrating that the fractional-order modeling approach leads to very parsimonious model formulations.
\end{abstract}

Index Terms-Analog circuit simulation, fractional-order systems, $R C$ circuits, system identification.

\section{INTRODUCTION}

$\mathbf{T}$ HE need for adopting fractional-order calculus in engineering stems from its ability to describe phenomena that cannot be fully described by a local theory. Examples include propagating acoustic or electromagnetic waves in the presence of boundaries, where, according to Huygens' principle, a propagating wave may be seen as a nonlocal process in space and time, inducing a memory effect (a delayed reaction) on a propagating wave [1], as well as when a smooth transition between a local (Newtonian) and a nonlocal (quantum) theory of motion is required. By incorporating nonlocal processes and phenomena in a mathematical framework, it becomes possible to extend the application of systems theory to a much wider range of problems across the physical sciences. The current contribution proposes an intuitive systems identification framework for complex systems with emergent behaviors emulated using a network of resistive and capacitive components, when

Manuscript received October 3, 2014; revised February 23, 2015 and March 23, 2015; accepted May 8, 2015. Date of publication May 21, 2015; date of current version August 28, 2015. This work was supported in part by Fundação de Amparo à Pesquisa do Estado de São Paulo under Grant 2011/ 17610-0 and Grant 2011/13777-8, by Conselho Nacional de Desenvolvimento Científico e Tecnológico (research fellowships), and by Coordenação de Aperfeiçoamento de Pessoal de Nível Superior (DINTER Program). This brief was recommended by Associate Editor E. Tlelo-Cuautle.

L. A. Jacyntho is with the Department of Mathematics, Universidade do Estado de Mato Grosso, 78390-000 Barra do Bugres-MT, Brazil (e-mail: luizantonioj@unemat.br).

M. C. M. Teixeira, E. Assunção, and R. Cardim are with the Department of Electrical Engineering, UNESP-Univ Estadual Paulista, 15385-000 Ilha Solteira-SP, Brazil (e-mail: marcelo@dee.feis.unesp.br; edvaldo@dee.feis. unesp.br; rcardim@dee.feis.unesp.br).

R. K. H. Galvão is with the Department of Electronic Engineering, Instituto Tecnológico de Aeronáutica, 12228-900 São José dos Campos-SP, Brazil (e-mail: kawakami@ita.br).

S. Hadjiloucas is with the School of Systems Engineering, University of Reading, Reading RG6 6AY, U.K. (e-mail: s.hadjiloucas@ reading.ac.uk).

Color versions of one or more of the figures in this brief are available online at http://ieeexplore.ieee.org.

Digital Object Identifier 10.1109/TCSII.2015.2436052

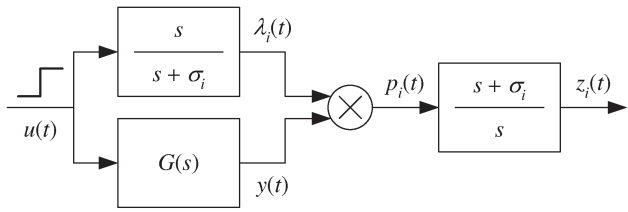

Fig. 1. Generation of the signals employed in the proposed identification procedure, using a given filtering parameter $\sigma_{i}>0$. The rectangular blocks correspond to transfer functions, whereas the circle with a $\times$ sign indicates a pointwise multiplication of the signals in the time domain.

these are subjected to a step excitation. The new algorithm aims to complement existing literature on the subject. Applications include filtering [2]-[10], analysis of dielectric responses [11]-[13], and control [14]-[18].

\section{A. Notation}

The Laplace transform of a signal $y(t), t \geq 0$ will be denoted by $Y(s)=\mathcal{L}[y(t)]$. The notation $\mathcal{L}^{-1}$ will indicate the inverse transform. Definitions will be stated by using the $\triangleq$ symbol. The limit values of a signal $y(t)$ will be denoted by $y\left(0^{+}\right) \triangleq \lim _{t \rightarrow 0^{+}} y(t), y(\infty) \triangleq \lim _{t \rightarrow \infty} y(t)$.

\section{Proposed Identification Method}

It is assumed that the system under consideration is stable and can be described by a transfer function of the form

$$
G(s)=\frac{b_{0}+b_{1} s^{\beta_{1}}+\cdots+b_{m} s^{\beta_{m}}}{1+a_{1} s^{\alpha_{1}}+\cdots+a_{n} s^{\alpha_{n}}}
$$

where $b_{0}, b_{1}, \ldots, b_{m}, a_{1}, \ldots, a_{n}$ are coefficients to be identified, and $\beta_{1}, \ldots, \beta_{m}, \alpha_{1}, \ldots, \alpha_{n}$ are positive real-valued exponents. Initially, these exponents will be assumed to be known.

The proposed identification method is based on the step response of the system, which is used to generate a set of auxiliary signals, as depicted in Fig. 1.

In Fig. 1, $y(t)$ represents the response of the system to a unit step $u(t), t \geq 0$, such that

$$
U(s)=1 / s, \quad Y(s)=G(s) U(s)=G(s) / s .
$$

In addition, $\lambda_{i}(t)$ is the result of passing the step input $u(t)$ through a filter with transfer function $s /\left(s+\sigma_{i}\right)$, i.e.,

$\lambda_{i}(t) \triangleq \mathcal{L}^{-1}\left[\frac{s}{s+\sigma_{i}} U(s)\right]=\mathcal{L}^{-1}\left[\frac{1}{s+\sigma_{i}}\right]=e^{-\sigma_{i} t}, \quad t \geq 0$

where $\sigma_{i}>0$ is a given filtering parameter. Signal $p_{i}(t)$ is obtained as $p_{i}(t) \triangleq y(t) \lambda_{i}(t)=y(t) e^{-\sigma_{i} t}, t \geq 0$, which corresponds to the following Laplace transform [19]:

$$
P_{i}(s)=\mathcal{L}\left[y(t) e^{-\sigma_{i} t}\right]=Y\left(s+\sigma_{i}\right) .
$$


From (2) and (4), it follows that

$$
P_{i}(s)=\frac{G\left(s+\sigma_{i}\right)}{s+\sigma_{i}} .
$$

Finally, $z_{i}(t), t \geq 0$ is the result of passing $p_{i}(t)$ through a filter with transfer function $\left(s+\sigma_{i}\right) / s$, i.e.,

$$
Z_{i}(s) \triangleq \frac{s+\sigma_{i}}{s} P_{i}(s)=\frac{G\left(s+\sigma_{i}\right)}{s} .
$$

In view of the final value theorem, which is also valid for fractional calculus [20], $z_{i}(\infty)$ can be related to $Z_{i}(s)$ as $z_{i}(\infty)=\lim _{s \rightarrow 0} s Z_{i}(s)$. By using this relation together with (1) and (6), it follows that

$$
z_{i}(\infty)=\lim _{s \rightarrow 0} G\left(s+\sigma_{i}\right)=\frac{b_{0}+b_{1} \sigma_{i}^{\beta_{1}}+\cdots+b_{m} \sigma_{i}^{\beta_{m}}}{1+a_{1} \sigma_{i}^{\alpha_{1}}+\cdots+a_{n} \sigma_{i}^{\alpha_{n}}}
$$

which can be rewritten as

$$
\begin{aligned}
z_{i}(\infty) a_{1} \sigma_{i}^{\alpha_{1}} & +\cdots+z_{i}(\infty) a_{n} \sigma_{i}^{\alpha_{n}} \\
& -b_{0}-b_{1} \sigma_{i}^{\beta_{1}}-\cdots-b_{m} \sigma_{i}^{\beta_{m}}=-z_{i}(\infty) .
\end{aligned}
$$

By evaluating $z_{i}(\infty)$ with $n+m+1$ different values of $\sigma_{i}$ $(i=1,2, \ldots, n+m+1),(8)$ can be used to derive a system of linear equations of the form $A \theta=c$, with (9) and (10), shown at the bottom of the page. Therefore, if $\operatorname{det}(A) \neq 0$, the model coefficients can be obtained by calculating $\theta=A^{-1} c$.

To conclude the presentation of the proposed method, there remain the issues of choosing appropriate values for the filtering parameters $\sigma_{1}, \sigma_{2}, \ldots, \sigma_{n+m+1}$ employed in the identification procedure and determining the transfer function exponents $\beta_{1}, \ldots, \beta_{m}, \alpha_{1}, \ldots, \alpha_{n}$.

For simplicity, the filtering parameters can be chosen as $\sigma_{2}=\rho \sigma_{1}, \sigma_{3}=\rho \sigma_{2}, \ldots, \sigma_{n+m+1}=\rho \sigma_{n+m}$, where $\rho$ is a constant in the range $0<\rho<1$. It is worth noting that the largest parameter $\sigma_{1}$ corresponds to the exponential function in (3) with fastest decay. In this brief, the value of $\sigma_{1}$ is adjusted to match the delay times of the exponential $e^{-\sigma_{1} t}$ and the step response $y(t)$. In this context, the delay time $t_{d}$ is defined as the time required for a function to reach the midpoint between its initial and final values, i.e., $y\left(t_{d}\right)=\left[y(\infty)+y\left(0^{+}\right)\right] / 2$. Therefore, $\sigma_{1}$ can be calculated as $\sigma_{1}=(\ln 2) / t_{d}$.

Finally, let $\gamma \triangleq\left[\begin{array}{llllll}\beta_{1} & \cdots & \beta_{m} & \alpha_{1} & \cdots & \alpha_{n}\end{array}\right]^{T}$ denote the vector of exponents to be determined. For given values of $\gamma$ and $\rho$, the identification procedure can be carried out in order to obtain the vector of transfer function coefficients as $\theta=A^{-1} c$. The following cost function $J(\gamma, \rho)$ can be then calculated as the root-mean-square error between the step response $y(t)$ and the output $\hat{y}(t)$ of the resulting model:

$$
J(\gamma, \rho) \triangleq \sqrt{\frac{1}{N} \sum_{k=1}^{N}[y(k T)-\hat{y}(k T)]^{2}}
$$

where $T$ is the sampling period employed in the acquisition of the step response, and $N$ is the number of acquired samples. Therefore, suitable values of $\gamma$ and $\rho$ can be obtained by using numerical optimization techniques to minimize $J(\gamma, \rho)$.

In this work, the FOTF MATLAB Toolbox [21] was employed to evaluate the step response $\hat{y}(t)$ of the fractional-order model, and the flexible polyhedron algorithm [22] implemented in the MATLAB Optimization Toolbox was adopted to obtain $\gamma$ and $\rho$ through the minimization of $J(\gamma, \rho)$.

Remark 1: To simplify the optimization procedure involved in the determination of the transfer function exponents, the following particularization can be adopted, as in [23]:

$$
\begin{array}{llrl}
\beta_{1} & =1, \ldots, \beta_{m-1}=(m-1), & & \beta_{m}=\beta \\
\alpha_{1} & =1, \ldots, \alpha_{n-1}=(n-1), & & \alpha_{n}=\alpha
\end{array}
$$

where $\beta$ and $\alpha$ will be the only real-valued exponents to be determined. Suitable values for $n$ and $m$ can be chosen by using parsimony criteria on the basis of the resulting approximation errors, as in integer-order identification [24].

Remark 2: A limitation of the proposed method is the need to choose a suitable value of $\rho$. It may be argued that this parameter affects the distribution of the approximation error over different frequencies. Indeed, larger values of $\rho$ result in the use of exponential functions with faster decay, which may lead to greater emphasis on the reduction of error at higher frequencies. This relation could be exploited to guide the selection of $\rho$, as an alternative to tuning this parameter through numerical optimization. Such a possibility will be pursued in future studies. Additional research will be also required for the analytical or the numerical derivation of uncertainty bounds on the identified coefficients and exponents.

Remark 3: Sufficient theoretical conditions for the invertibility of the matrix $A$ defined in (9) still need to be investigated. However, no singularity or ill-conditioning problems were experienced in this work.

\section{Case Study: Three-Dimensional RC Network}

Fig. 2 presents an example of the RC networks under consideration, which can be used as circuit models for the dielectric response of composite materials containing insulating and

$$
\begin{aligned}
& A=\left[\begin{array}{ccccccc}
z_{1}(\infty) \sigma_{1}^{\alpha_{1}} & \cdots & z_{1}(\infty) \sigma_{1}^{\alpha_{n}} & -1 & -\sigma_{1}^{\beta_{1}} & \cdots & -\sigma_{1}^{\beta_{m}} \\
z_{2}(\infty) \sigma_{2}^{\alpha_{1}} & \cdots & z_{2}(\infty) \sigma_{2}^{\alpha_{n}} & -1 & -\sigma_{2}^{\beta_{1}} & \cdots & -\sigma_{2}^{\beta_{m}} \\
\vdots & \vdots & \vdots & \vdots & \vdots & \vdots & \vdots \\
z_{n+m+1}(\infty) \sigma_{n+m+1}^{\alpha_{1}} & \cdots & z_{n+m+1}(\infty) \sigma_{n+m+1}^{\alpha_{n}} & -1 & -\sigma_{n+m+1}^{\beta_{1}} & \cdots & -\sigma_{n+m+1}^{\beta_{m}}
\end{array}\right] \\
& \theta=\left[a_{1} \cdots a_{n} b_{0} b_{1} \cdots b_{m}\right]^{T}, \quad c=\left[\begin{array}{c}
-z_{1}(\infty) \\
-z_{2}(\infty) \\
\vdots \\
-z_{n+m+1}(\infty)
\end{array}\right]
\end{aligned}
$$




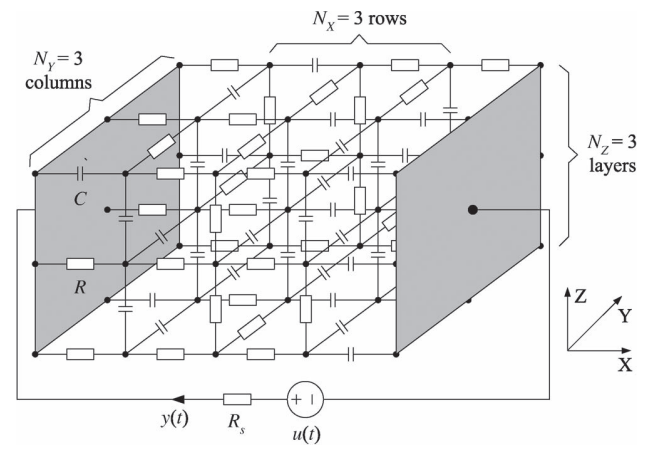

Fig. 2. Diagram of a 3-D RC network with random allocation of the $R, C$ elements. The gray plates indicate electrodes employed for connection to a voltage source with output resistance $R_{S}$.

conducting particles [12]. The topology consists of $N_{Z}$ layers, each consisting of a matrix of nodes with $N_{X}$ rows and $N_{Y}$ columns. A resistor $R_{S}$ is included to model the internal resistance of the source. The numbers of resistors (not including the source) and capacitors are denoted by $N_{R}$ and $N_{C}$, respectively, so that $N_{R}+N_{C}=N_{Z}\left[N_{Y}\left(N_{X}+1\right)+N_{X}\left(N_{Y}-\right.\right.$ 1)] $+\left(N_{Z}-1\right) N_{X} N_{Y}$ [23]. The fraction of capacitors is defined as $f_{C}=N_{C} /\left(N_{R}+N_{C}\right)$.

As shown in [23], a dynamic model in descriptor form can be derived from Kirchoff's laws to relate the input voltage $u(t)$ with the current $y(t)$ entering the network. A randomized incidence matrix can be employed to place the $R, C$ components at aleatory positions. The resulting model can be converted to an integer-order transfer function $G(s)$, which facilitates the timedomain simulation of the network, as well as the calculation of the admittance in the frequency domain.

It is worth noting that the model order can be very high, even after taking into account algebraic constraints associated to closed paths of capacitors and eliminating noncontrollable or nonobservable modes. For example, the network employed in [23], with 100 resistors and 100 capacitors, resulted in a transfer function with order 73. However, it was found that a more compact representation could be obtained by using a fractionalorder transfer function of the form

$$
G_{\text {frac }}(s)=\frac{b_{0}+b_{1} s+\cdots+b_{n-1} s^{n-1}+b_{n} s^{\beta}}{1+a_{1} s+\cdots+a_{n-1} s^{n-1}+a_{n} s^{\alpha}}
$$

where single fractional exponents $\alpha, \beta$ are employed at the denominator and numerator, as stated in (12).

The identification procedure adopted in [23] was aimed at matching the exact admittance $G(j \omega)$ of the network and the approximated admittance $G_{\text {frac }}(j \omega)$ in fractional-order form, over a given range of frequencies $\omega$. For this purpose, the coefficients $b_{0}, b_{1}, \ldots, b_{n}, a_{1}, \ldots, a_{n}$ and the fractional exponents $\alpha, \beta$ were adjusted to minimize the following cost:

$$
J=\sum_{k=1}^{N} w_{k}\left|G\left(j \omega_{k}\right)-G_{\text {frac }}\left(j \omega_{k}\right)\right|^{2}
$$

where $\omega_{1}, \omega_{2}, \ldots, \omega_{N}$ are frequencies of interest chosen by the designer, and $w_{1}, w_{2}, \ldots, w_{N}>0$ are frequency-dependent weights. The flexible polyhedron algorithm [22] was employed for the minimization of $J$ with respect to the coefficients and fractional exponents. As a result, a transfer function $G_{\text {frac }}(s)$ with $n=2$, involving only seven parameters $\left(b_{0}, b_{1}, b_{2}, a_{1}, a_{2}\right.$,
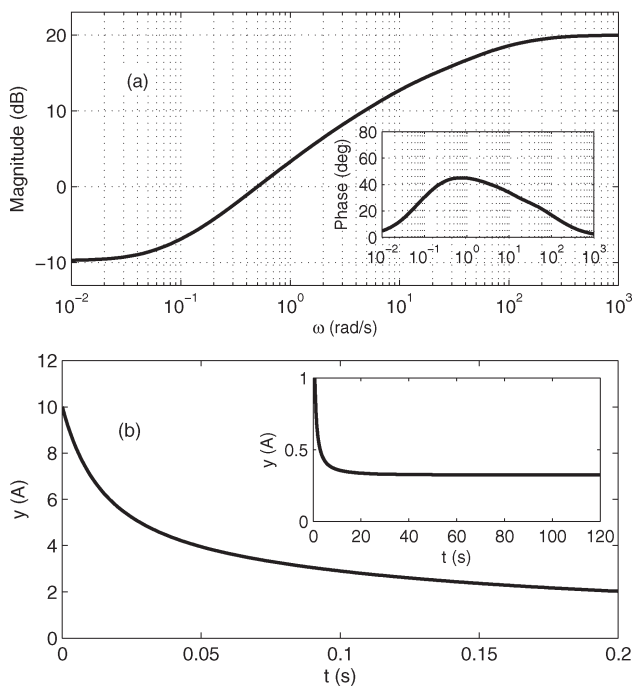

Fig. 3. (a) Admittance and (b) unit step response of the RC network.

$\beta, \alpha$ ), was sufficient to provide a good approximation of the admittance over a broad frequency range.

In what follows, the results of the proposed identification method will be compared with those reported in [23], which were obtained by minimizing the cost (14) over $N=500$ frequencies logarithmically spaced between $\omega_{1}=10^{-2} \mathrm{rad} / \mathrm{s}$ and $\omega_{500}=10^{3} \mathrm{rad} / \mathrm{s}$. The weights were set to $w_{k}=\left|G\left(j \omega_{k}\right)\right|^{-2}$ in order to normalize the cost [23]. The same network will be employed, with parameters $R_{S}=0.1 \Omega, R=1 \Omega, C=0.5 \mathrm{~F}$, $N_{Z}=3, N_{X}=N_{Y}=5$, and $f_{C}=0.5$.

\section{RESUlTS}

\section{A. Preliminary Analysis of the RC Network Response}

Fig. 3(a) and (b) presents the admittance and unit step response of the network, including the source resistance $R_{S}$. As shown in Fig. 3(a), the admittance converges to $-10 \mathrm{~dB}$ at low frequencies, which amounts to a resistance of $3.16 \Omega$. This value corresponds to the association of $R_{S}$ with the equivalent resistance of the RC network, since the capacitors behave as open circuits at low frequencies. As a result, the steady-state value of the input current following the $1-\mathrm{V}$ step excitation is equal to $1 \mathrm{~V} / 3.16 \Omega=0.32 \mathrm{~A}$, which is consistent with the limit value of the curve shown in the inset in Fig. 3(b).

The convergence of the admittance to $+20 \mathrm{~dB}$ at high frequencies can be ascribed to a path of capacitors between the network terminals. Indeed, since the capacitors behave as short circuits at high frequencies, the overall admittance becomes $20 \log _{10}\left(1 / R_{S}\right)=20 \log _{10}(1 / 0.1)=20 \mathrm{~dB}$. This is also the reason why the input current reaches an initial value of $10 \mathrm{~A}$ in Fig. 3(b). The capacitors impose that the voltage across the network remains equal to zero immediately after the application of the $1-\mathrm{V}$ step excitation, and thus, the initial current is equal to $1 \mathrm{~V} / R_{S}=1 \mathrm{~V} / 0.1 \Omega=10 \mathrm{~A}$.

\section{B. Identification Results}

The proposed identification method was initially employed with a fractional-order transfer function of the form

$$
G_{\text {frac }}^{n=1}(s)=\frac{b_{0}+b_{1} s^{\alpha}}{1+a_{1} s^{\alpha}}
$$


TABLE I

SUMMARY OF RESULTS

\begin{tabular}{lcc}
\hline & $n=1$ & $n=2$ \\
\hline (a) & $\frac{1.449 s+0.358}{0.229 s+1}$ & $\frac{0.875 s^{2}+2.73 s+0.339}{0.116 s^{2}+1.614 s+1}$ \\
(b) $\frac{1.348 s^{0.682}+0.287}{0.145 s^{0.682}+1}$ & $\frac{5.311 s+0.792 s^{0.439}+0.333}{0.481 s+2.994 s^{0.439}+1}$ \\
(c) $\frac{1.395 s^{0.698}+0.281}{0.181 s^{0.651}+1}$ & $\frac{0.067 s+3.015 s^{0.852}+0.346}{0.088 s+1.288 s^{0.472}+1}$
\end{tabular}

(a) Proposed method (integer order), (b) Proposed method (fractional order), (c) Results reported in [23] (Fractional order).

where $\alpha>0$ is a real-valued exponent. This is a particular case of (13), with $n=1$, in which the same fractional exponent is used in the numerator and denominator. This configuration was adopted in view of the asymptotic behavior of the network admittance at high frequencies, as discussed earlier. Therefore, the cost (11) becomes a function of only two parameters, namely, $\alpha$ and $\rho$. In view of the possible convergence to poor local minima, the optimization was carried out from different initial values of $(\alpha, \rho)$ in a grid formed by varying $\alpha$ from 0.1 to 1.0 and $\rho$ from 0.1 to 0.9 , both with a step size of 0.1 .

The proposed method was also employed to identify a fractional-order transfer function of the form

$$
G_{\text {frac }}^{n=2}(s)=\frac{b_{0}+b_{1} s+b_{2} s^{\alpha}}{1+a_{1} s+a_{2} s^{\alpha}}
$$

which is a particular case of (13) with $n=2$ and the same fractional exponent $\alpha$ in the numerator and denominator. The initial $(\alpha, \rho)$ pairs employed in the optimization were taken from a grid formed by varying $\alpha$ from 0.1 to 2.0 and $\rho$ from 0.1 to 0.9 , both with a step size of 0.1 . For comparison, the following integer-order transfer functions were also identified:

$$
G_{\text {int }}^{n=1}(s)=\frac{b_{0}+b_{1} s}{1+a_{1} s}, \quad G_{\text {int }}^{n=2}(s)=\frac{b_{0}+b_{1} s+b_{2} s^{2}}{1+a_{1} s+a_{2} s^{2}} .
$$

In this case, the cost (11) becomes a function of a single parameter $\rho$. This parameter was optimized by using initial values ranging from 0.1 to 0.9 , with a step size of 0.1 .

The identification was based on the step response of the RC network, with a time range of $0-80 \mathrm{~s}$ and sampling period $T=$ $0.001 \mathrm{~s}$. By using a computer with an i5 processor $(1.70 \mathrm{GHz})$, the optimization times for each initial $(\alpha, \rho)$ point were approximately 3 and $11 \mathrm{~min}$ for the integer- and fractional-order models, respectively. No substantial differences between $n=1$ and $n=2$ were observed. The workload is mainly associated to the generation of the model output $\hat{y}(k T)$ employed in the cost function (11). The computational demand of such a procedure is much larger in the fractional-order case.

Table I presents the resulting integer- and fractional-order transfer functions, along with the results reported in [23].

Fig. 4 compares the step responses of the integer- and fractional-order models obtained with the proposed method. As can be seen, the fractional-order models provide a much better match of the network response. For $n=2$, the model response is almost indistinguishable from that of the network.

Fig. 5 compares the step response of the fractional-order model obtained here with that reported in [23], which are labeled "step response identification" and "frequency response identification," respectively. The comparison is restricted to $n=2$, because the transfer function obtained in [23] for $n=1$
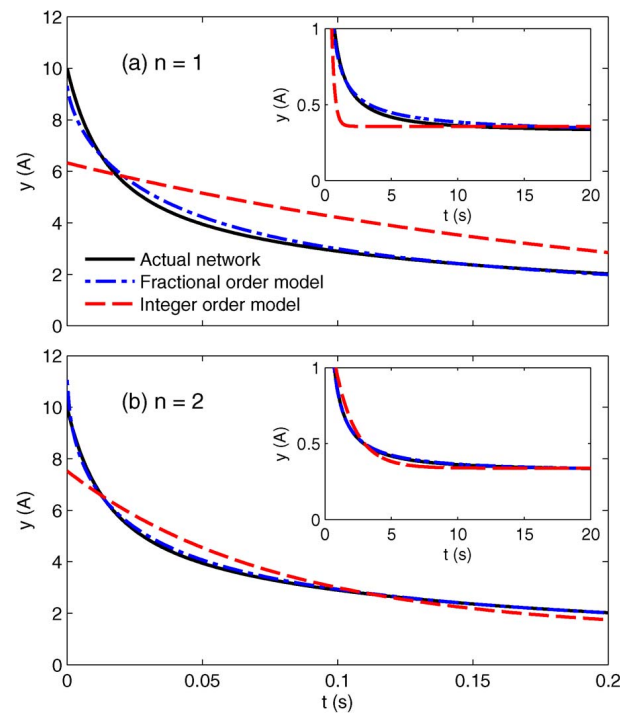

Fig. 4. Step response results: Fractional- and integer-order models.

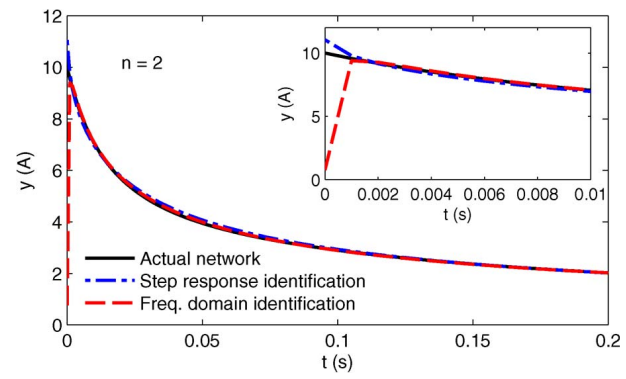

Fig. 5. Step response results (fractional-order model with $n=2$ ): Comparison with the frequency-domain identification method reported in [23].

is not proper (i.e., the dominating term in the numerator has a larger exponent compared with the denominator), as shown in Table I. Therefore, the corresponding step response is not well defined. In fact, the parametrization adopted in [23] did not impose that the fractional exponents should be equal in the numerator and denominator. Since the identification was aimed at matching the network within a limited frequency range, there was no need to impose an a priori relation between these exponents. With regard to the $n=2$ case in Fig. 5 , it can be seen that both models provide a good match of the network response. However, the model reported in [23] yields a larger discrepancy in the initial part of the transient response, as shown in the inset. This finding may be ascribed to a mismatch between the admittance of the network and the identified model at frequencies higher than those employed in the cost function (14). Since the method proposed here is directly aimed at matching the step response, the resulting model provides a much better match of the initial response.

A dramatic improvement using the fractional-order over the integral-order formulation is shown in Fig. 6, which corroborates the time-domain findings. Finally, Fig. 7 compares the frequency responses of the fractional-order models obtained here with those reported in [23]. For $n=2$, the results show excellent agreement to the network frequency response and are consistent with those in [23]. The proposed method, tailored at matching the step response in the time domain, also provides an excellent match in the frequency domain. 

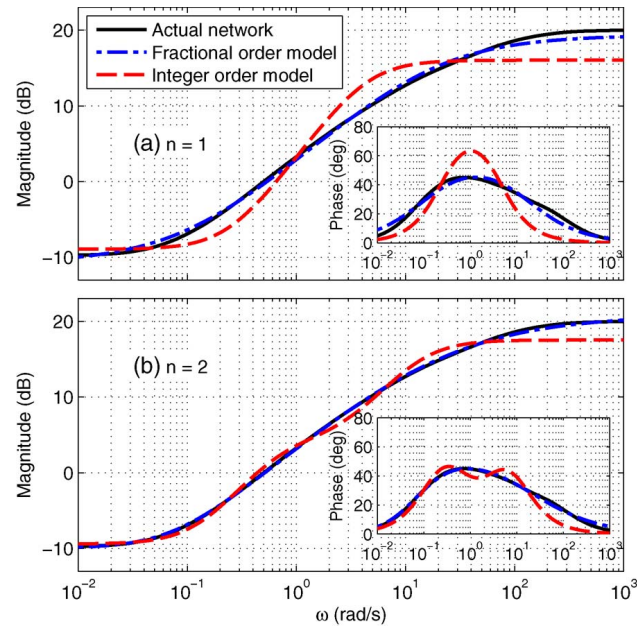

Fig. 6. Frequency-domain results: Fractional- and integer-order models.
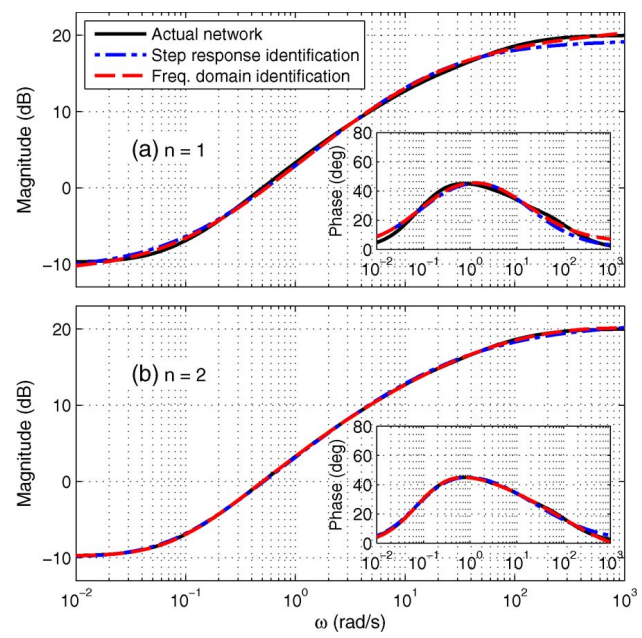

Fig. 7. Frequency-domain results (fractional-order models): Comparison with the frequency-domain identification method reported in [23].

\section{CONCLUSION}

This brief has discussed the identification of fractionalorder transfer functions using a step excitation. The main advantage of this formulation is that fewer parameters need to be numerically optimized. Instead of optimizing the coefficients and fractional exponents of the transfer function, the proposed method only requires the optimization of the fractional exponents, as well as the parameter $\rho$ employed in the definition of the auxiliary filtering parameters. Algorithmic implementation is straightforward using existing computational toolboxes.

Fractional-order systems were generated in software using a nodal analysis of 3-D RC circuits. These networks can be tailored in terms of topology and composition to emulate real time systems governed by fractional-order dynamics. A single fractional exponent $\alpha$ was sufficient to obtain an excellent matching of the network dynamics in the time and frequency domains. Therefore, only two parameters $(\alpha$ and $\rho$ ) had to be numerically optimized. The proposed step response identification procedure has a very parsimonious formulation, matches very well the network step response, and is consistent with previous frequency-domain formulations [23].

\section{REFERENCES}

[1] N. Engheta, "Fractional paradigm in electromagnetic theory," in Frontiers in Electromagnetics, D. H. Werner and R. Mittra, Eds. New York, NY, USA: IEEE Press, 2000, ch. 12.

[2] G. Carlson and C. Halijak, "Approximation of fractional capacitors $(1 / s)^{1} / n$ by a regular Newton process," IEEE Trans. Circuit Theory, vol. CT-11, no. 2, pp. 210-213, Jun. 1964.

[3] S. Samadi, M. O. Ahmad, and M. N. S. Swamy, "Results on maximally flat fractional delay systems," IEEE Trans. Circuits Syst. I, vol. 51, no. 11, pp. 2271-2286, Nov. 2004.

[4] R. S. Barbosa, J. A. T. Machado, and M. F. Silva, "Time domain design of fractional differintegrators using least-squares," Signal Process., vol. 86, no. 10 , pp. 2567-2581, Oct. 2006.

[5] A. Oustaloup, F. Levron, B. Mathieu, and F. M. Nanot, "Frequencyband complex noninteger differentiator: Characterization and synthesis," IEEE Trans. Circuits Syst. I, vol. CAS1-47, no. 1, pp. $25-39,2000$.

[6] C.-C. Tseng, "Design of fractional order digital FIR differentiators," IEEE Signal Process. Lett., vol. 8, no. 3, pp. 77-79, Mar. 2001.

[7] H. H. Dam, "Variable fractional delay FIR filter design with a bicriteria and coefficient relationship," IEEE Trans. Circuits Syst. II, vol. 61, no. 1, pp. 36-40, Jan. 2014.

[8] H. Johansson, "Fractional-delay and supersymmetric Mth-band linearphase FIR filters utilizing partially symmetric and antisymmetric impulse responses," IEEE Trans. Circuits Syst. II, vol. 59, no. 6, pp. 366-370, Jun. 2012.

[9] G. Maione, "Closed-form rational approximations of fractional, analog and digital differentiators/integrators," IEEE J. Emerging Sel. Topics Circuits Syst., vol. 3, no. 3, pp. 322-329, Sep. 2013.

[10] D. Chen, Y. Q. Chen, and D. Xue, "Digital fractional order SavitzkyGolay differentiator," IEEE Trans. Circuits Syst. II, vol. 58, no. 11, pp. 758-762, Nov. 2011.

[11] R. Bouamrane and D. P. Almond, "The emergent scaling phenomenon and the dielectric properties of random resistor-capacitor networks," J. Phys., Condensed Matter, vol. 15, no. 24, pp. 4089-4100, 2003.

[12] N. J. McCullen, D. P. Almond, C. J. Budd, and G. W. Hunt, "The robustness of the emergent scaling property of random RC network models of complex materials," J. Phys. D, Appl. Phys., vol. 42, no. 6, 2009, Art. ID. 064001.

[13] R. K. H. Galvão et al., "Multivariate analysis of random threedimensional RC networks in the time and frequency domains," IEEE Trans. Dielectr. Electr. Insulat., vol. 20, no. 3, pp. 995-1008, Jun. 2013.

[14] I. Podlubny, I. Petrás, B. M. Vinagre, P. O’Leary, and L. Dorcák, "Analogue realizations of fractional-order controllers," Nonlinear Dynam., vol. 29, no. 1-4, pp. 281-296, Jul. 2002.

[15] B. M. Vinagre, I. Podlubny, A. Hernandez, and V. Feliu, "Some approximations of fractional order operators used in control theory and applications," J. Fractional Calculus Appl. Anal., vol. 3, no. 3, pp. 231-248, 2000.

[16] L. Chen, Y. Chai, R. Wu, and J. Yang, "Stability and stabilization of a class of nonlinear fractional-order systems with Caputo derivative," IEEE Trans. Circuits Syst. II, vol. 59, no. 9, pp. 602-606, Sep. 2012.

[17] C.-C. Hua, D. Liu, and X.-P. Guan, "Necessary and sufficient stability criteria for a class of fractional-order delayed systems," IEEE Trans. Circuits Syst. II, vol. 61, no. 1, pp. 59-63, Jan. 2014.

[18] M. A. Pakzad and M. A. Nekoui, "Stability analysis of linear timeinvariant fractional exponential delay systems," IEEE Trans. Circuits Syst. II, vol. 61, no. 9, pp. 721-725, Sep. 2014.

[19] G. F. Franklin, J. D. Powell, and A. Emami-Naeini, Feedback Control of Dynamic Systems, 5th ed. Upper Saddle River, NJ, USA: PrenticeHall, 2006.

[20] C. P. Li, W. H. Deng, and D. Xu, "Chaos synchronization of the Chua system with a fractional order," Physica A, vol. 360, no. 2, pp. 171-185, Feb. 2006.

[21] Y. Q. Chen, I. Petras, and D. Xue, "Fractional order control—A tutorial," in Proc. Amer. Control Conf., St. Louis, MO, USA, 2009, pp. 1397-1411.

[22] P. E. Gill, W. Murray, and M. H. Wright, Practical Optimization. New York, NY, USA: Academic, 1981

[23] R. K. H. Galvão, S. Hadjiloucas, K. H. Kienitz, H. M. Paiva, and R. J. M. Afonso, "Fractional order modeling of large three-dimensional RC networks," IEEE Trans. Circuits Syst. I, vol. 60, no. 3, pp. 624-637, Mar. 2013.

[24] L. Ljung, System Identification. Upper Saddle River, NJ, USA: Prentice-Hall, 1999. 